\title{
Towards rotation sensing with a single atomic clock
}

\author{
Thomas Fernholz*a ${ }^{\mathrm{a}}$, Robin Stevenson ${ }^{\mathrm{a}}$, Michael R. Hush ${ }^{\mathrm{b}}$, Igor W. Lesanovsky ${ }^{\mathrm{a}}$, Thomas Bishop ${ }^{\mathrm{a}}$, \\ Fabio Gentile $^{\mathrm{a}}$, Sindhu Jammi ${ }^{\mathrm{a}}$, Tadas Pyragius ${ }^{\mathrm{a}}$, Mark G. Bason ${ }^{\mathrm{a}}$, \\ Hèctor Mas ${ }^{c, d}$, Saurabh Pandey ${ }^{c, e}$, Georgios Vasilakis ${ }^{c}$, Konstantinos Poulios ${ }^{c}$, and Wolf von \\ Klitzing ${ }^{\mathrm{c}}$ \\ ${ }^{a}$ School of Physics \& Astronomy, The University of Nottingham, University Park, Nottingham NG7 2RD, UK; \\ ${ }^{\mathrm{b}} \mathrm{School}$ of Engineering and Information Technology, University of New South Wales at the Australian Defence Force \\ Academy, Canberra 2600, Australia; \\ ' Institute of Electronic Structure and Laser, Foundation for Research and Technology - Hellas, Heraklion 70013, \\ Greece; ${ }^{\mathrm{d}}$ Department of Physics, University of Crete, Heraklion 70113, Greece; \\ ${ }^{\mathrm{e}}$ Department of Materials, Science and Technology, University of Crete, Heraklion 70113, Greece
}

\begin{abstract}
We discuss a scheme to implement a gyroscopic atom sensor with magnetically trapped ultra-cold atoms. Unlike standard light or matter wave Sagnac interferometers no free wave propagation is used. Interferometer operation is controlled only with static, radio-frequency and microwave magnetic fields, which removes the need for interferometric stability of optical laser beams. Due to the confinement of atoms, the scheme may allow the construction of small scale portable sensors. We discuss the main elements of the scheme and report on recent results and efforts towards its experimental realization.
\end{abstract}

Keywords: Atomic sensor, Sagnac interferometer, cold atom interferometry, navigation sensor, ring-shaped atom traps, radio-frequency dressed potentials, TAAP.

\section{INTRODUCTION}

The Sagnac effect originally describes the consequences of rotation on the path difference between two light beams allowed to propagate around a closed loop. The most sensitive gyroscopes are those based on this effect and have technological applications, such as inertial sensing ${ }^{1}$, seismology ${ }^{2}$ and also in fundamental physics ${ }^{3}$.

It was argued by Clauser ${ }^{4}$ that using atomic matter waves instead of visible light leads to an intrinsic enhancement of sensitivity by 11 orders of magnitude as it scales with the mass of the interfering particles. While this comparison is optimistic due to much smaller achievable particle flux in matter waves than in light waves, impressive advances have been made in recent decades, see recent review ${ }^{5}$. High performance with sensitivities down to the range of $10^{-9} \mathrm{rad} \mathrm{s}^{-1} / \sqrt{\mathrm{Hz}}$ was achieved with atoms in free fall ${ }^{6,7}$. Since the available cycle time for free fall experiments and thus the enclosed interferometer area is limited by the apparatus size, there are now considerable development efforts towards guided interferometers, where the propagation of atoms is confined to a loop.

\section{MEASURING THE SAGNAC EFFECT}

\subsection{Sagnac effect as propertime difference}

The task of a gyroscope is to measure the rotational frequency $\Omega$ of an apparatus or observational frame, e.g., a vehicle or a laboratory that co-rotates with Earth. The Sagnac effect is associated with interferometry in such rotating frames and can be described in terms of the propagation time difference between two waves that are sent around a loop of radius $R$ in opposite directions. As seen from an inertial frame, a relative phase shift will be introduced because the sourcedetector assembly will have moved due to rotation of the setup before the waves recombine. The situation as seen from the laboratory frame can be explained using the theory of relativity. For matter waves a simple description can be given in the two oppositely rotating frames of co-moving observers. If a rotating experimenter launches particles from an 
*thomas.fernholz@nottingham.ac.uk; phone +44 115 9514753;

www.nottingham.ac.uk/Physics/research/coldatomsgroup/home.aspx

angular position $\theta=0$ at such speed that they recombine at the opposite side of the loop of radius $R$ after time $T$, the recombination will occur at $\theta=\pi+\Omega T$ as judged by an inertial observer. In the inertial frame, the particles will therefore travel at different average angular speeds $\omega_{+/-}=\pi / T \pm \Omega$. According to special relativity, two co-moving observers will therefore measure propertimes between launch and recombination that differ for small speeds by $\Delta \tau \approx 2 \pi \Omega \mathrm{R}^{2} / c^{2}$, independent of the duration $T$. This relativistic effect was experimentally confirmed by Hafele and Keating, by comparing atomic clocks flown around Earth where the time difference amounts to a few hundred nanoseconds, depending on the path [8]. For particles of mass $m$ in an interferometer, their quantum state can be described in their respective rest frame as evolving at the Compton frequency $\omega_{C}=m c^{2} / \hbar$. Upon recombination, the particles will thus acquire a relative phase shift of by $\Delta\left(\omega_{C} \tau\right) \approx \omega_{C} \Delta \tau+\Delta \omega_{C} \tau$ for non-relativistic speeds. The first term in this difference equals the propagation phase difference in the inertial frame and results in the Sagnac phase shift for a half-revolution of $\Delta \varphi=\omega_{C} \Delta \tau=2 \pi \Omega \mathrm{R}^{2} \mathrm{~m} / \hbar$. The second term $\Delta \omega_{C} \tau \approx \Delta E T / \hbar$ accounts for differences in relativistic mass or energy $\Delta E$, which includes the difference in internal energy for atoms in different clock states. It is the second term that is responsible for the Hafele-Keating result, where the dynamically accumulated phase between atoms in clock states of almost identical relativistic energy was used to determine the individual propertimes of the two paths.

\subsection{A single clock scheme}

The measurement with two atomic clocks makes use of four different quantum states, two pairs of internal states travelling along the same paths. But atoms are only put into superpositions of co-propagating states, namely a superposition of internal states travelling clockwise and a superposition of internal states travelling anti-clockwise. In contrast, a Sagnac interferometer measures the phase difference between only two, counter-propagating states, which may be linked to different internal states, e.g., when using optical Raman beam splitting. It is important to note, however, that the Sagnac effect does not rely on the free propagation of any waves. It can also be measured by transporting trapped atoms in opposite directions.

We recently discussed such a scheme that uses magnetically trappable clock states, which can be found in the hyperfine ground state manifolds of alkali atoms ${ }^{9,10}$. The interferometric sequence is depicted in Fig. 1. A ring-shaped potential is deformed such that a state-independent harmonic trap forms at $\theta=0$, which is initially loaded with atoms in state $\mid \downarrow)$. A $\pi / 2$-pulse driven on the clock transition prepares atoms in a superposition $|\psi\rangle=(|\downarrow\rangle+|\uparrow\rangle) / \sqrt{2}$. After state-dependent transport to the opposite side of the ring-shaped path, a $\pi$-pulse at time of duration $T$ can be used to exchange populations in the states $|\uparrow\rangle$ and $|\downarrow\rangle$ in order to achieve common path operation. This also removes the dynamical clock phase as the atoms spend half the observer's time in each internal state. Transport is continued to complete a full revolution after which a final $\pi / 2$-pulse at time $2 T$ converts the accumulated Sagnac phase into population difference.
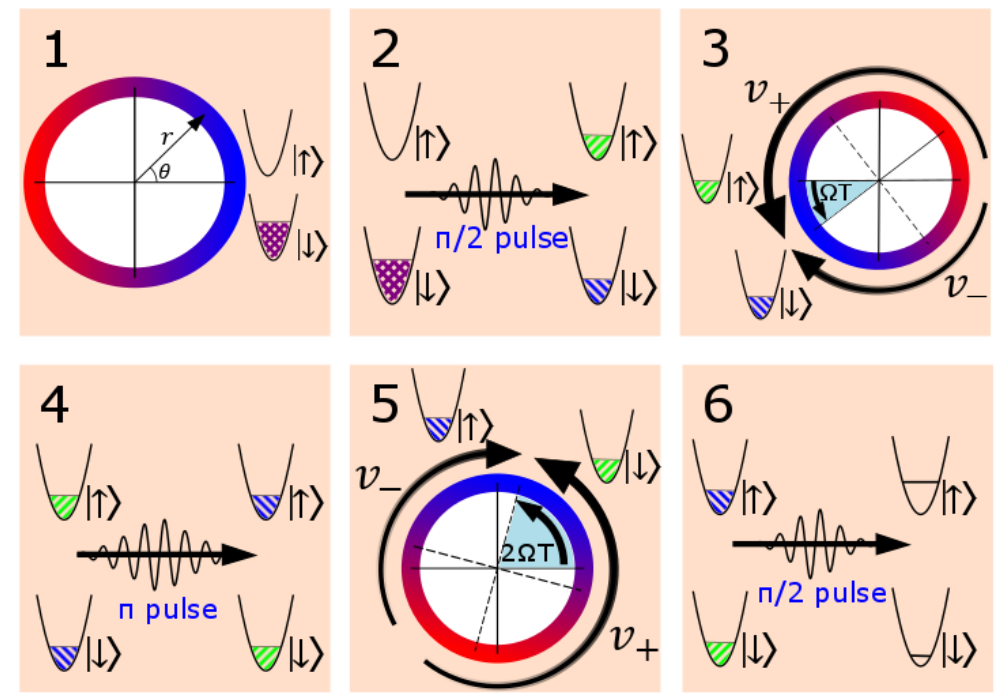
Figure 1. Ramsey sequence for a clock based Sagnac interferometer rotating at frequency $\Omega$ with state-dependent transport.

Due to the rotation of the laboratory frame, the traps are transported at different speeds $v_{+}$- as seen from an inertial frame.

\subsection{Dynamical effects and interferometer contrast}

We analyzed such a fully guided scheme theoretically for simplified trapping potentials in one-dimensional (infinite radial and axial confinement) and two-dimensionally spherical harmonic traps ${ }^{10}$. This allowed us to investigate some dynamical effects that affect the interferometer performance with non-interacting ground-state and thermal atoms.

In the one-dimensional case, the interferometer will always measure the Sagnac phase, but with potentially reduced contrast. The operation is essentially that of a white-light interferometer where the final overlap of the two wavepackets must be on the order of the coherence length of the thermal gas. Any spatial separation as well as non-zero relative momentum between the two counter-propagating ensembles during the final $\pi / 2$-pulse will lead to contrast reduction. Acceleration and deceleration of the traps during the transport must not result in motional excitation at the end of the sequence. The quantity that determines the contrast is the coherent displacement $\Delta a$ between the two harmonic oscillators of frequency $a$ at the end of the sequence. The contrast at temperature $\Theta$ is given by $C=\exp \left(-\left(1 / 2+k_{B} \Theta / \hbar \omega\right)|\Delta \alpha|^{2}\right)$. For symmetrically transported traps, the final displacement is a function of the temporal angular speed profile $\Omega_{\text {path }}(t)$ of the two traps as seen in the laboratory frame and given by $\Delta \alpha=R \sqrt{2 m \omega / \hbar} \int_{0}^{2 T} \Omega_{\text {path }}(t) \exp (-i \omega t) d t$. If the speed profile does not contain Fourier components at the trapping frequency, full contrast will be achieved.

Further effects can influence the interferometer performance, e.g. if the radial confinement is finite. The sensitivity of the interferometer can be reduced due to contrast reduction of final wave packet overlap but may also be increased or decreased further due to three different effects. The first effect is that centrifugal forces lead to increased path radius and enclosed interferometer area. This does not only depend on the transport profile but also on the laboratory rotation $\Omega$. Centrifugal forces may in principle even overcome radial harmonic confinement. The second effect occurs for non-zero laboratory rotation, where the centrifugal forces will differ in the two paths. In this situation atoms will acquire phase difference due to different radial dynamics and potential energies in their respective traps. Depending on the procedure to extract laboratory rotation from the interferometric signal, the third effect may influence the sensitivity to rotation because not only the Sagnac phase but also the contrast depend on the laboratory rotation. The analytical description of the combined effects ${ }^{10}$ requires numerical integration for arbitrary temporal speed profiles.

\section{STATE-DEPENDENT, RADIO-FREQUENCY DRESSED POTENTIALS}

In the following we discuss how radio-frequency dressed potentials can be used to generate a variety of geometries, including ring-shaped traps, and discuss state-dependence of such potentials.

\subsection{Radio-frequency dressed potentials}

The basic idea of radio-frequency dressed potentials is not very different from standard magnetic trapping in which cold, thus slowly moving atoms, adiabatically maintain the relative direction of their spin with respect to a spatially dependent magnetic field. When atoms, e.g., traverse the minimum of a Ioffe-Pritchard type trap orthogonally to the trap axis, their spin and consequently the direction of force adiabatically reverses direction, and atoms remain in the trapped state. In radio-frequency dressed traps this necessary change in spin-direction is induced by adiabatic passage across a resonance between Larmor frequency and an oscillating field.

If we neglect internal structure, which leads to higher order Zeeman effects, the time-dependent interaction Hamiltonian of an atom with spin $F$ in a magnetic field with static and oscillatory components is given by

$$
\widehat{H}=\mu_{B} g_{F} \widehat{\boldsymbol{F}} \cdot\left(\boldsymbol{B}_{D C}+\boldsymbol{B}_{R F}(\omega t)\right),
$$

Where $\mu_{B}$ is the Bohr magneton, $g_{F}$ is the Landé Factor, and the momentum operator is expressed in units of $\hbar$. The oscillating part can be best expressed in terms of spherical polarization components. Using the spherical basis $\boldsymbol{e}_{ \pm}=\left(\boldsymbol{e}_{x} \pm i \boldsymbol{e}_{y}\right) / \sqrt{2}, \boldsymbol{e}_{\pi}=\boldsymbol{e}_{z}$, with a $Z$-axis chosen in the direction of the static field such that $\boldsymbol{B}_{D C}=B_{D C} \boldsymbol{e}_{z}$, we can write

$$
\boldsymbol{B}_{R F}(\omega t)=\operatorname{Re}\left[\left(B_{+} \boldsymbol{e}_{+}+B_{-} \boldsymbol{e}_{-}+B_{\pi} \boldsymbol{e}_{\pi}\right) \times e^{i \omega t}\right]
$$


Using the corresponding spin components with the conventional normalization of raising and lowering operators $\widehat{\boldsymbol{F}}_{ \pm}=\widehat{\boldsymbol{F}}_{x} \pm i \widehat{\boldsymbol{F}}_{\gamma}$, the Hamiltonian becomes

$$
\widehat{H}=\frac{1}{2} \mu_{B} g_{F}\left[B_{D C} \hat{F}_{Z}+\left(\frac{1}{\sqrt{2}} B_{+} \hat{F}_{+}+\frac{1}{\sqrt{2}} B_{-} \hat{F}_{-}+B_{\pi} \hat{F}_{Z}\right) \times e^{i \omega t}\right]+c_{.} c_{\nu}
$$

Transforming to a frame rotating about the $z$-axis at frequency $a$, i.e. using $\widehat{H}_{\text {rot }}=i \hbar \frac{\partial}{\partial t} \widehat{U} \widehat{U}-1+\widehat{U} \widehat{H} \widehat{U}^{-1}$ with $\widehat{U}=e^{-i F_{z} \omega t}$ and the identity $e^{\alpha F_{z}} \hat{F}_{ \pm} e^{-\alpha F_{Z}}=e^{ \pm \alpha} \widehat{F}_{ \pm}$, partially removes the time-dependence of the Hamiltonian

$$
\widehat{H}_{\text {rot }}=\frac{1}{2} \mu_{B} g_{F}\left[\left(B_{D C}-B_{\text {res }}\right) \hat{F}_{z}+\left(\frac{1}{\sqrt{2}} B_{+} \hat{F}_{+}+\frac{1}{\sqrt{2}} B \cdot \hat{F} \times e^{2 i \omega t}+B_{\pi} \hat{F}_{z} \times e^{i \omega t}\right)\right]+c . c .,
$$

where we introduced the resonant field $B_{\text {res }}=\hbar \omega / \mu_{B} g_{F}$. If the RF field is polarized purely in the $\boldsymbol{e}_{+}$direction, i.e., $B_{-}=B_{\pi}=0$, atoms are subject to the same potential and behavior as in an apparently static, effective field $\boldsymbol{B}_{\text {eff }}=\frac{1}{2 \sqrt{2}}\left(B_{+} \boldsymbol{e}_{+}+\right.$c.c. $)+\left(B_{D C}-B_{\text {res }}\right) \boldsymbol{e}_{z}$. This field may exhibit a minimum, typically near locations where $B_{D C}=B_{\text {res }}$, and atomic spin will adiabatically follow its orientation provided that reorientation occurs much slower than the effective Larmor frequency. When other RF-polarization components are present, the rotating wave approximation can be used, thus neglecting the fast oscillating terms ${ }^{11}$ of $\widehat{H}_{r o t}$ as long as $|\hbar \omega| \gg \mu_{B}\left|g_{F} \boldsymbol{B}_{\text {eff }}\right|$. The resulting potential remains the same apart from second order energy shifts ${ }^{12}$. It is important to note that for negative $g_{F}$ the frequency $a$ must be negative for a positive resonant field. This inverts the sense of rotation that is required to dress atoms with negative Landé factor.

\subsection{State-dependence in ring shaped potentials}

We identified two configurations which result in ring-shaped potentials that can be used to introduce state-dependent guiding within these potentials. The first potential is based on a dressed ring-shaped quadrupole field, whereas the second uses time averaging to create a ring-shaped trap from a dressed 3D quadrupole field.

\subsection{The dressed quadrupole ring}

The first arrangement uses an axially symmetric field with two-dimensional quadrupole character in the vicinity of a ring of zero field. Such a configuration can be generated by four circular currents, e.g., using two-stacked pairs of concentric currents as shown in Fig. 2.
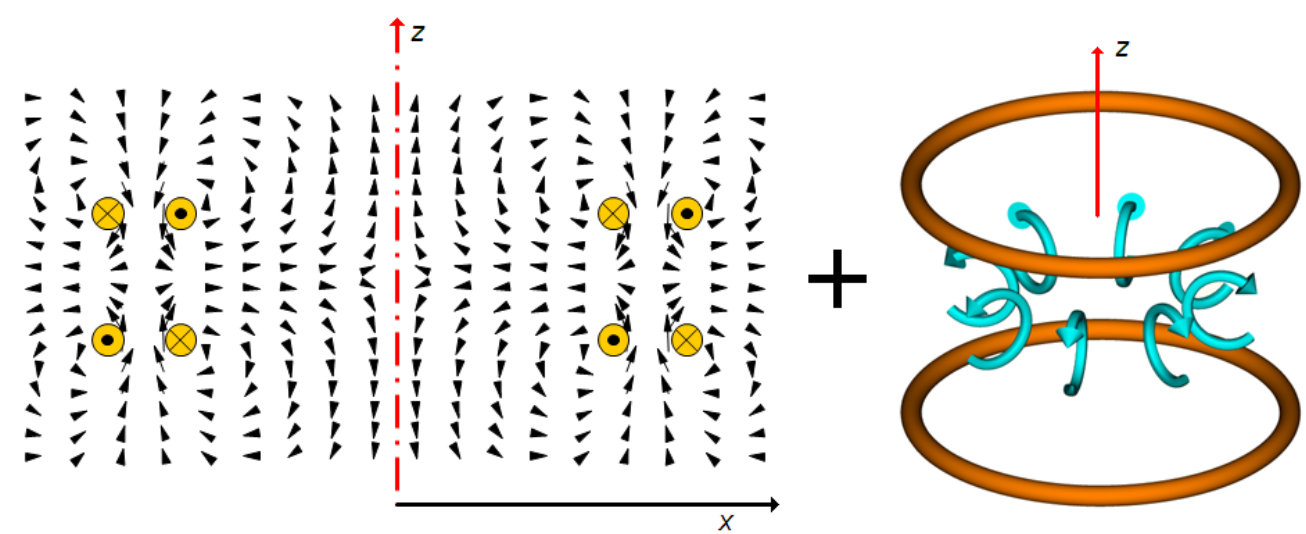

Figure 2. Field configuration for the dressed quadrupole ring. An axially symmetric field with a ring of zero field strength is generated by four circular currents (left). The dressing RF field is a combination of Helmholtz and anti-Helmholtz character with $90^{\circ}$ phase difference (right).

Atoms in this static field will be dressed by a superposition of two RF fields, a homogenous axial field and a $90^{\circ}$ out-ofphase radial field. This can be generated by a pair of co-axial coils placed symmetrically around the static trap. The result is an axially symmetric dressing field that is circularly or elliptically polarized near the static quadrupole ring. We have previously calculated idealized potentials resulting from this configuration. By choice of parameters, and including additional radial fields, the possibilities range from forming a hollow torus to stacked or concentric rings to singlyconnected traps, which become state-dependent ${ }^{9}$. 
A depiction of the results is shown in Fig. 3. An overall potential minimum is formed near the surface of a hollow torus where the atomic Larmor frequency that is determined by the modulus of the static magnetic field is resonant with the used radio-frequency. If the RF field near this torus is circularly polarized, its $\boldsymbol{e}_{+}$-component and thus the potential minimum are constant over the surface of the torus, see Equ. 4. For elliptical polarization, the decomposition into spherical components varies in the poloidal direction, which results in two rings of minimal potential.

The potential within the rings can further be altered by additional RF fields. If the static field underlying the rings points in the axial direction, an RF field that is circularly polarized in the toroidal plane will result in additional $\boldsymbol{e}_{+}$components. As these interfere with the decomposition of the primary RF field, potential minima will occur within the rings whose position in the toroidal direction is determined by the relative phase between the two RF fields.

The key feature of this configuration is that the primary setup is fully axially symmetric and leads to equal amounts of $\boldsymbol{e}_{+^{-}}$and $\boldsymbol{e}_{-}$-polarization in the decomposition into spherical RF field components. The potential thus depends only on the modulus of the Landé-factor. A field polarized in the toroidal plane will break this symmetry and states with oppositely signed Landé-factors can be controlled independently. In fact, ramping the phase of a single in-plane linearly polarized field, i.e., an equal combination of $\sigma^{+}$- and $\sigma^{-}$-polarisation with respect to the axial direction, will lead to the desired state-dependent behavior that is required to operate a Sagnac interferometer.

If the magnetically trappable clock states of rubidium- 87 are used ${ }^{13}$, the state-dependent trapping potentials can be made nearly identical in confinement and sensitivity to external field fluctuations. An additional advantage of this scheme is the simultaneous operation of two oppositely oriented interferometers. This is due to the fact that the static fields underlying the two rings will necessarily point in opposite directions and the state-dependence reverses direction ${ }^{9}$. In this way, two closely spaced interferometers can be operated simultaneously and a differential measurement will cancel a range of external influences, including phase noise from the required microwave reference that drives the clock transition.

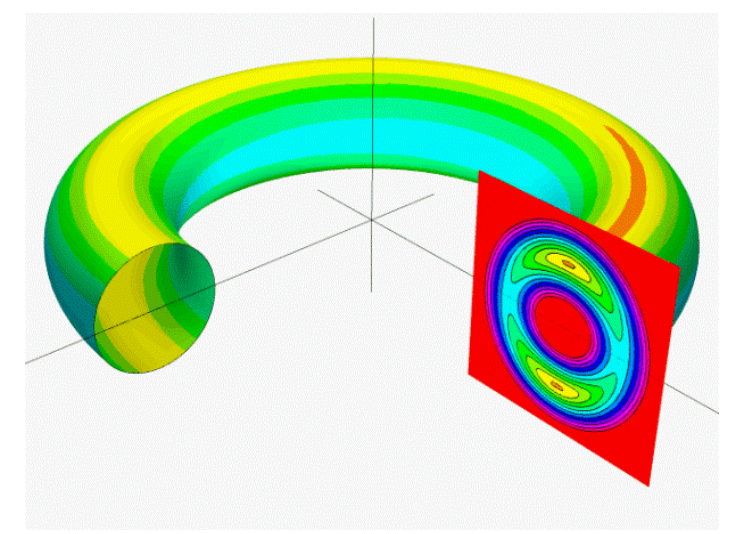

Figure 3. State-dependent RF-dressed potential in idealized field configuration. Atoms will be trapped in a toroidal geometry, with the possibility to introduce ring-shaped as well as toroidally localized potential minima by control of RFpolarization.

\subsection{Time averaged adiabatic potentials}

Time averaged adiabatic potential (TAAP) rings are formed from a combination of a static quadrupole field with rfdressing of atoms and a homogeneous oscillating field. The behavior of atoms with respect to an external electromagnetic field can be understood by considering the dynamics on three different time scales ${ }^{14}$. Radio-frequency dressing occurs on the fastest time scale. The internal atomic spin states adiabatically change as the atoms traverse regions where their Larmor frequency is resonant with the RF. On a medium time scale, alterations of the adiabatic potential experienced by the atoms that are fast enough to leave the center of mass motion of atoms unaffected results in a timeaveraged potential. On the slowest time scale, the motional states of atoms will also adiabatically follow changes of the time averaged potential. 
Conceptually, we start with a static 3D-quadrupole field with radial field gradient $\alpha$, see Fig. 4.), which is dressed by a strong RF-field at frequency $\omega_{R F}$ and maximal Rabi frequency $\Omega_{R F}$. The resulting potential is minimal near points of resonance. As the resonant Larmor frequency is determined by the static field strength, a trap in the form of an oblate ellipsoid is formed. The potential within this trap varies depending on the orientation of the dressing field with respect to the local static field.

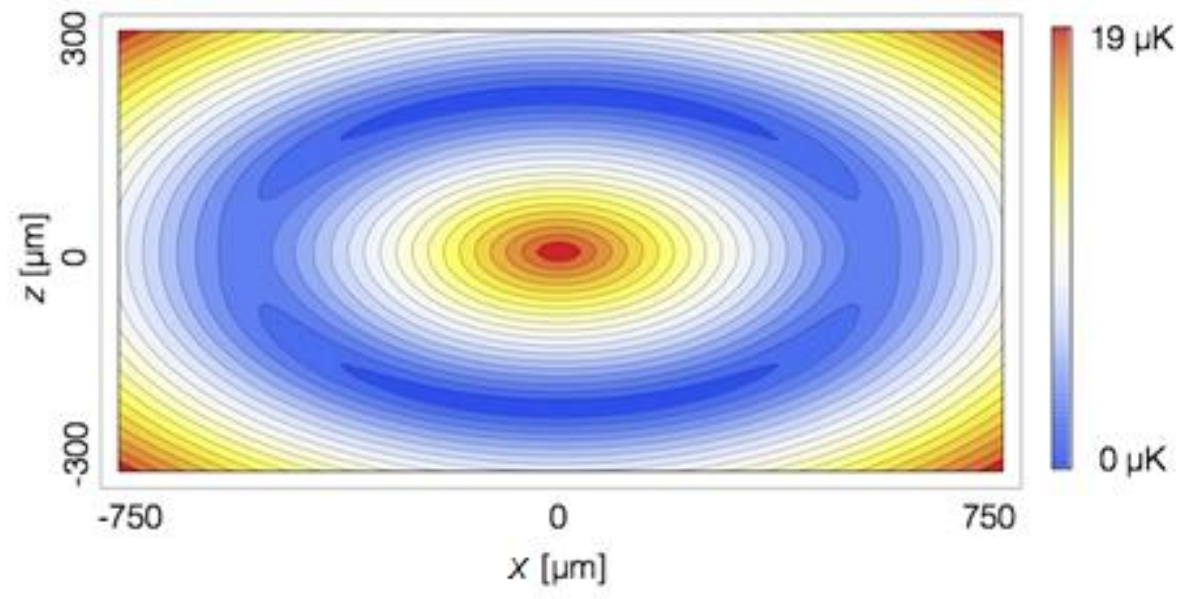

Figure 4. Contour plot of a vertical cut through the potential resulting from a quadrupole field dressed by an RF-field oscillating parallel to the $\mathrm{z}$-axis. Note that due to the decomposition into spherical polarization components of the RF, the

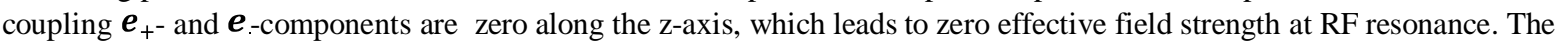
plot parameters are $\omega_{R F}=2 \pi \times 2.5 \mathrm{MHz}$, maximal RF Rabi frequency of $2 \pi \times 2.5 \mathrm{MHz}$ and $30 \mathrm{G} / \mathrm{cm}$ static field gradient.

The potential shown in Fig. 4 is modified by a time-averaging, homogenous, vertically polarized field $B_{m}$, which oscillates at a frequency that is small compared to the Larmor frequency in the resonance region. The effective potential results from averaging over the modulation cycle. The resulting ring-shaped potential including gravity is shown in Fig. 5.

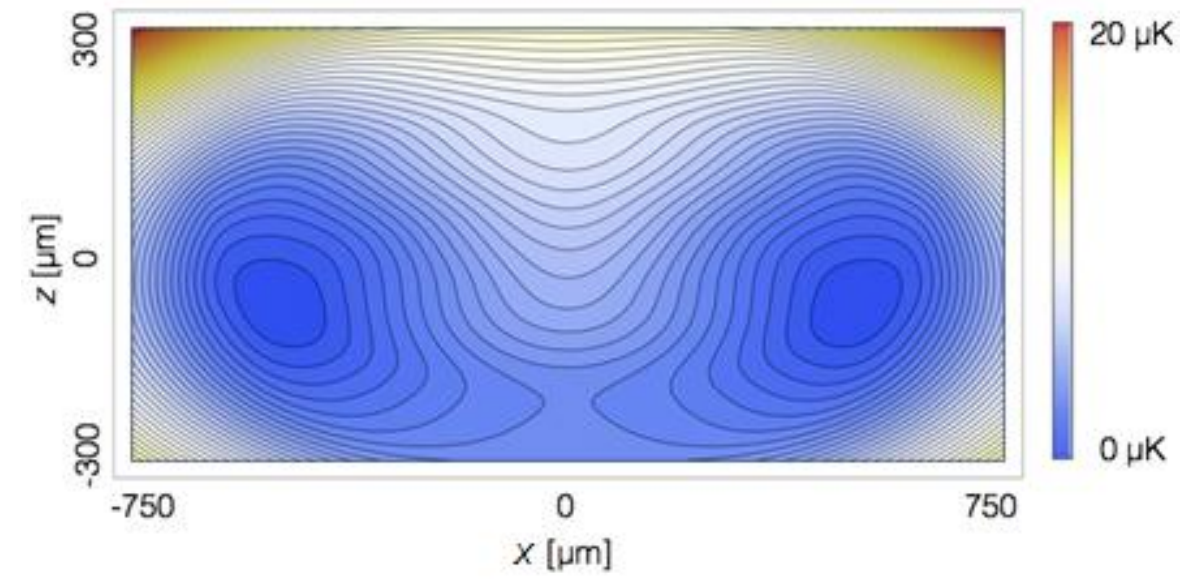

Figure 5. Contour plot of a vertical cut through the potential of a TAAP ring-trap including gravity. The parameters are identical to Fig. 4 with the addition of a vertical $1.3 \mathrm{G}$ modulation field.

Without gravity, the radius of the resulting ring is $\rho=g_{F} \mu_{B} \alpha / \hbar \omega_{R F}$. Under the simplifying assumption that the time averaging field does not affect the RF coupling on the ring, we can express the radial and vertical trapping frequencies $\omega_{\rho}$ and $\omega_{7}$ for an atom of mass $m$ trapped in the ring as ${ }^{14}$ 


$$
\begin{gathered}
\omega_{\rho}=\omega_{0}\left(1+\beta^{2}\right)^{-1 / 4}, \\
\omega_{z}=2 \omega_{0}\left\lceil 1-\left(1+\beta^{2}\right)^{-1 / 2}\right\rceil^{1 / 2},
\end{gathered}
$$

with $\omega_{0}=m_{\mathrm{F}} g_{\mathrm{F}} \mu_{\mathrm{B}} \alpha / \sqrt{m \hbar \Omega_{\mathrm{RF}}}$ and $\beta=g_{\mathrm{F}} \mu_{\mathrm{R}} B_{\mathrm{m}} / \hbar \omega_{\mathrm{RF}}$.

State-dependence of the trap can be achieved by adding a weak horizontal RF-field in the $y$-direction, which is 90 degrees out of phase with respect to the vertical oscillation. This creates a small circular component, which lies in the $y, z$-plane. Therefore, the coupling strength due to the resulting $\boldsymbol{e}_{+(-)}$-component is tilted along the $x$-direction, depending on $g_{F}$ and thus on the internal atomic state.
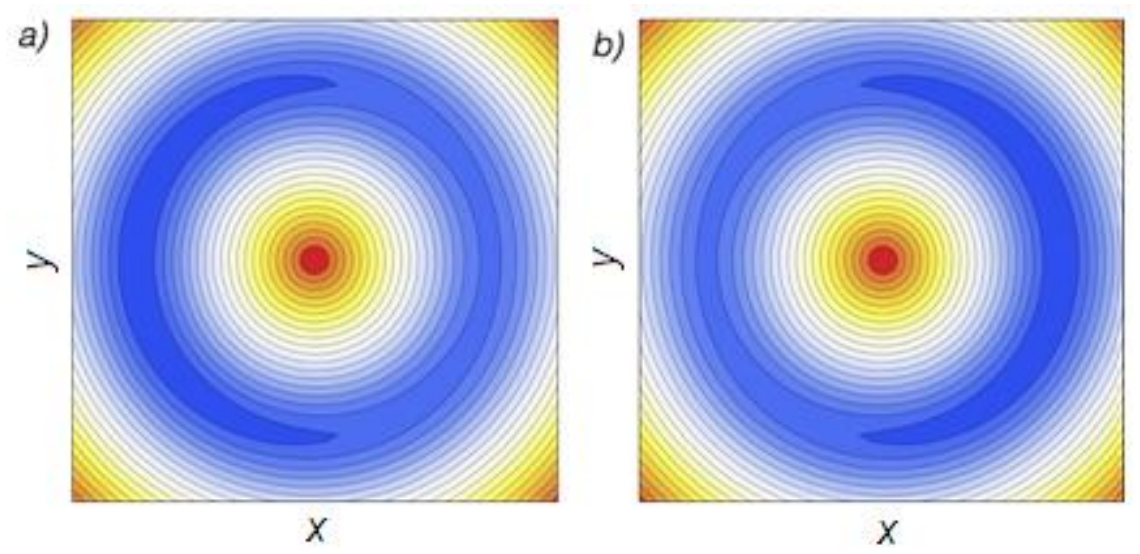

Figure 6. Contour plots of a horizontal cut through the potential of a TAAP ring-trap excluding gravity for rubidium-87 atoms in states $\left|F=1, m_{F}=-1\right\rangle$ (a) and $\left|F=2, m_{F}=+1\right\rangle$ (b).

\section{EXPERIMENTAL REALISATION}

\subsection{Towards a dual interferometer in a static quadrupole ring}

We are exploring several routes to generating and loading a radio-frequency dressed quadrupole trap with atoms. In view of portable devices, it may be beneficial to use permanent magnetic structures to generate the required static fields. Such structures can be used to reduce power consumption, reduce magnetic field noise and maintain the axial symmetry that would be broken by any lead-wires of electro-magnets.

One issue to address is the loading and cooling sequence of atoms into such a trap. It is possible to load atoms into permanent magnetic traps by applying a sequence of external fields that temporarily cancel the permanent fields along a loading trajectory. This method was already used to load a permanent magnetic Ioffe-Pritchard type trap and produce Bose-Einstein condensates ${ }^{15}$. It is, e.g., possible to use an axially magnetized ring magnet together with a variable axial field to continuously tune from a 3-dimensional quadrupole field used for a standard magneto-optical trap (MOT) to a quadrupole ring, see Fig. 7. Atoms can be laser-cooled and then magnetically trap and transformed into the ring. In fact, it is possible to operate a magneto-optical trap directly in a quadrupole ring ${ }^{16}$. A section of a ring-shaped MOT is shown in Fig. 8. 


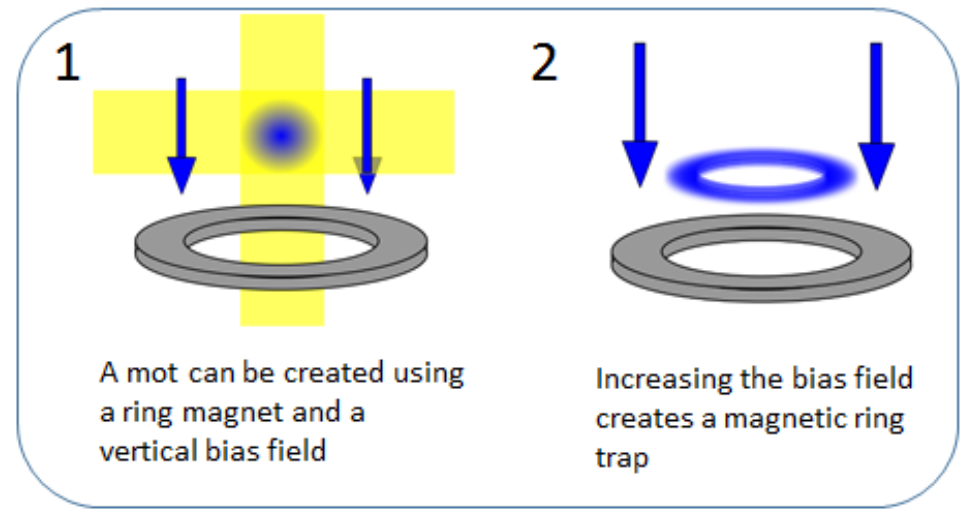

Figure 7. Loading a permanent magnetic quadrupole ring with laser-cooled atoms. The combination of permanent ring magnet with a variable axial field allows for the operation of a MOT as well as the generation of a quadrupole ring.

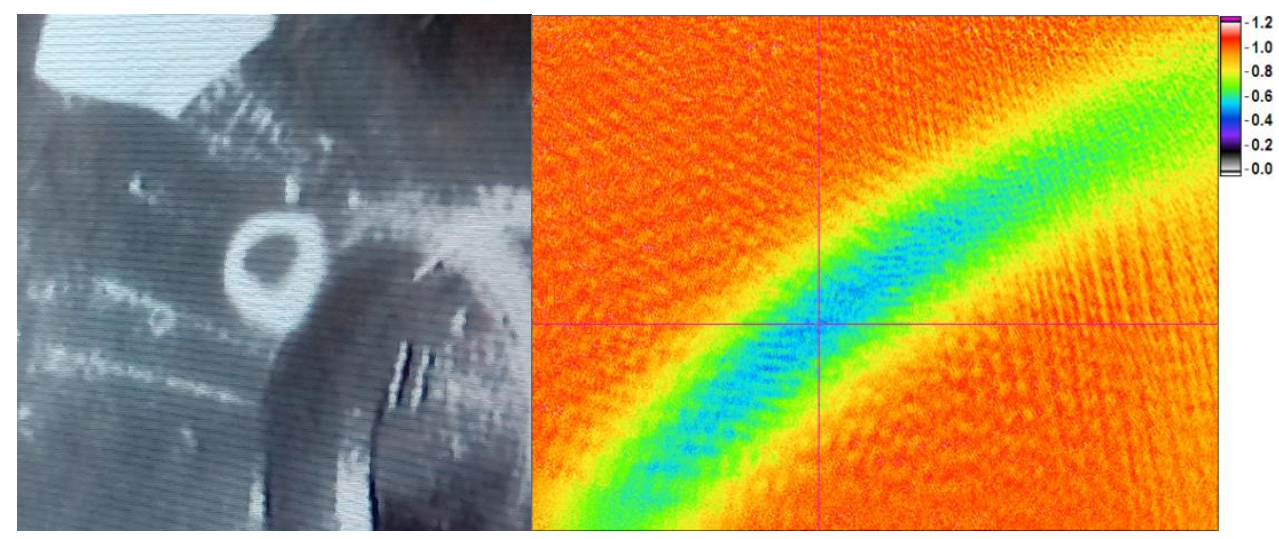

Figure 8 . Photograph and absorption image of atoms in a ring-shaped magneto-optical trap ${ }^{16}$. The necessary quadrupole ring was generated by cancelling the field of an permanent ring magnet with an external, homogenous, axial field.

In a similar fashion, it is possible to load a quadrupole ring that is formed without external fields. Such a ring is formed when two concentric rings of permanent magnetic material are used. Fig. 9 shows calculated contours of the resulting magnetic field produced from this configuration and external homogeneous and gradient fields. In this sequence, an initial ring-shaped trap is displaced and moved towards infinity while a second trap splits into the ring an eventually replaces the original. In this way it becomes possible to continuously compress atoms from an initial three-dimensional quadrupole trap into the final ring.

We are considering different sizes and techniques to evaluate effects that will arise, e.g., from atomic collisions, coherence times and confinement parameters. Small rings with diameters of a few hundred microns can be generated using micro-fabrication techniques. Using structures with ring thickness of around $100 \mu \mathrm{m}$ and magnetization of 1000 $\mathrm{emu} / \mathrm{cc}$ traps of around $30 \mu \mathrm{K}$ trap depth at a distance of around $50 \mu \mathrm{m}$ from the surface. 

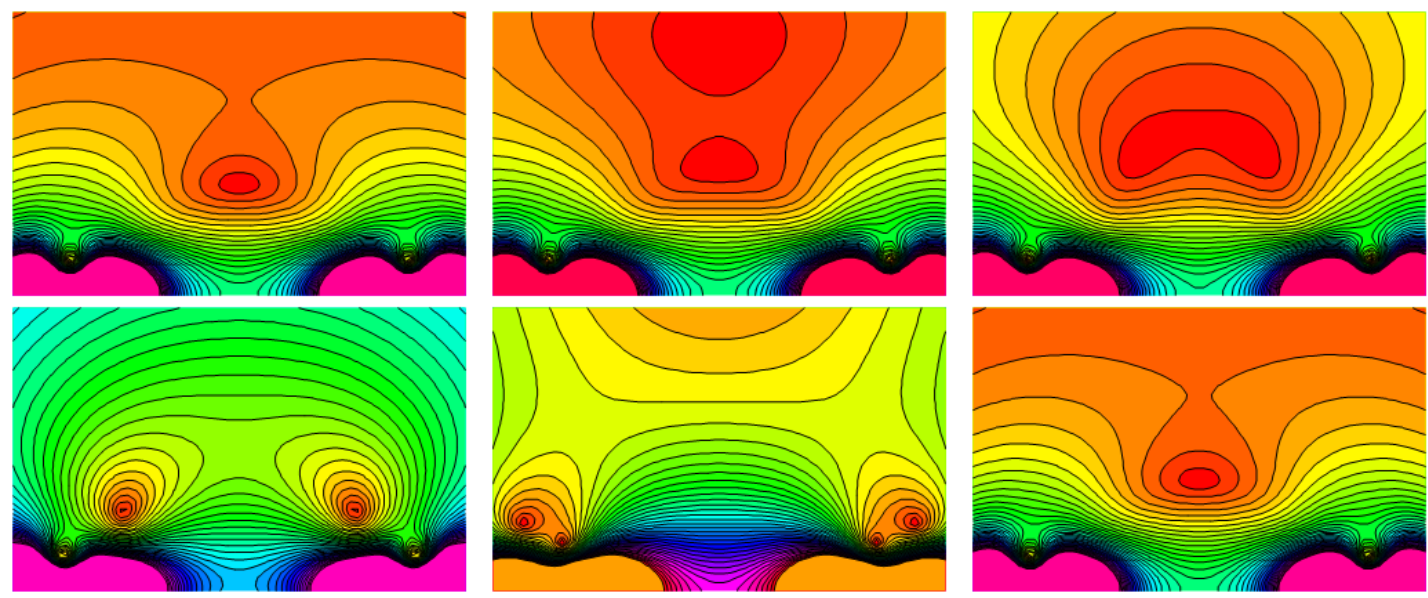

Figure 9. Loading sequence of a self-biased quadrupole ring. Two concentric rings of permanent magnetic material located at the bottom plane of the images produce a static quadrupole ring as well as a central three-dimensional quadrupole. By applying external fields it is possible to compress a distant magnetic trap, which joins with the central quadrupole to form a ring that eventually replaces the original trap.

\subsection{Recent results in TARP}

Here, we demonstrate the individual steps required for a clock type interferometer in rf-TAAP rings, notably the tapping and state-sensitive manipulation of atoms in rf-TAAP rings.

Our experimental setup for the generation of rf-TAAP rings consists of a double vacuum system with a 2D-MOT section and a 3D-MOT section. After loading the MOT for about $10 \mathrm{~s}$, we create a very cold atom cloud (or BEC) in a quadrupole TOP trap. We then ramp up a TOP field of $5.3 \mathrm{G}$ and switch on the dressing field at a frequency of $2.62 \mathrm{MHz}$ with a linear polarization along the z-axis. This frequency is chosen such that the fundamental and its first harmonic are equally distant from the Larmor frequency of the atoms at the center of the trap. We then ramp down the TOP amplitude, thus placing the atoms in a dumbbell-shaped trap with all the atoms being in the lower well. We then rotate the axis of the TOP modulation to move the atoms away from the z-axis. We then lower the TOP-field in the $\mathrm{x}$ - and $\mathrm{y}$ axes whilst increasing the field in the z-axis, resulting in the desired ring-shaped trap.

In order to achieve this, we need to have full 3D control of the amplitude and polarisation of both the modulation and radio frequency fields. We generate the main dressing rf using two horizontal single-loop coils in Helmholtz configuration ( $20 \mathrm{~mm}$ radius at a distance of $26 \mathrm{~mm}$ ) resulting in a homogeneous $\mathrm{rf}$ field, which is linearly polarized in the direction of the z-axis. In order to achieve the required field strengths of about $1 \mathrm{G}$ at a frequency $2.4 \mathrm{MHz}$, we connect a set of capacitors in parallel with the coils, thus generating a resonator with an on-resonance resistance of a few hundred ohms. The horizontal components of the rf field are generated by two overlapping pairs of rectangular coils are placed in the $\mathrm{x}$ and $\mathrm{y}$ directions again with capacitors in parallel. All three circuits are driven by $25 \mathrm{~W}$ amplifiers (AmplifierReserach model 25A250A or MiniCircuits LZY-22+). As a frequency source we use two two-channel Tektronix Function Generators (AFG3022) both linked to a Rubidium Frequency Standard (Stanford Research Instruments FS725).

The figure below shows about about $4.8 \times 10^{5}$ rubidium- 87 atoms in a flat ring-shaped waveguide. Here, we use a linear dressing field along the z-axis and a vertical modulation field at $2.8 \mathrm{G}$. Note that at the optical resolution no corrugations of the wave-guide can be detected, and that the structures visible in the plot are due to pixilation. 

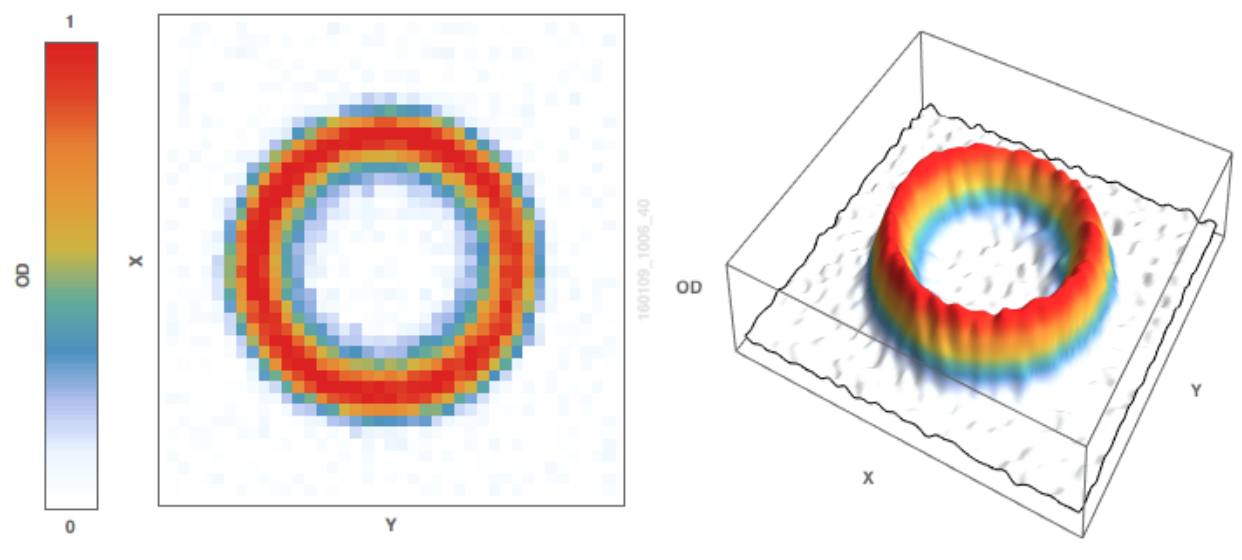

Figure 10. A TAAP ring with atoms in $\mathrm{F}=2, \mathrm{~m}_{\mathrm{F}}=2$ state, quadrupole gradient $\alpha=50 \mathrm{G} / \mathrm{cm}$, dressing frequency $\omega_{r f} / 2 \pi=$ $2.62 \mathrm{MHz}$ and radius of the ring is $570 \mu \mathrm{m}$. Atom number in the ring is about $4.8 \times 10^{5}$.

One great advantage of the rf-TAAP rings is that one can change the radius of the ring at will simply by adjusting the gradient of the quadrupole or the frequency of the dressing field. Using the first method we have demonstrated rings from $40 \mu \mathrm{m}$ to $2.4 \mathrm{~mm}$ in diameter. Figure 11 shows the variation of the radius of the ring versus the quadrupole gradient.

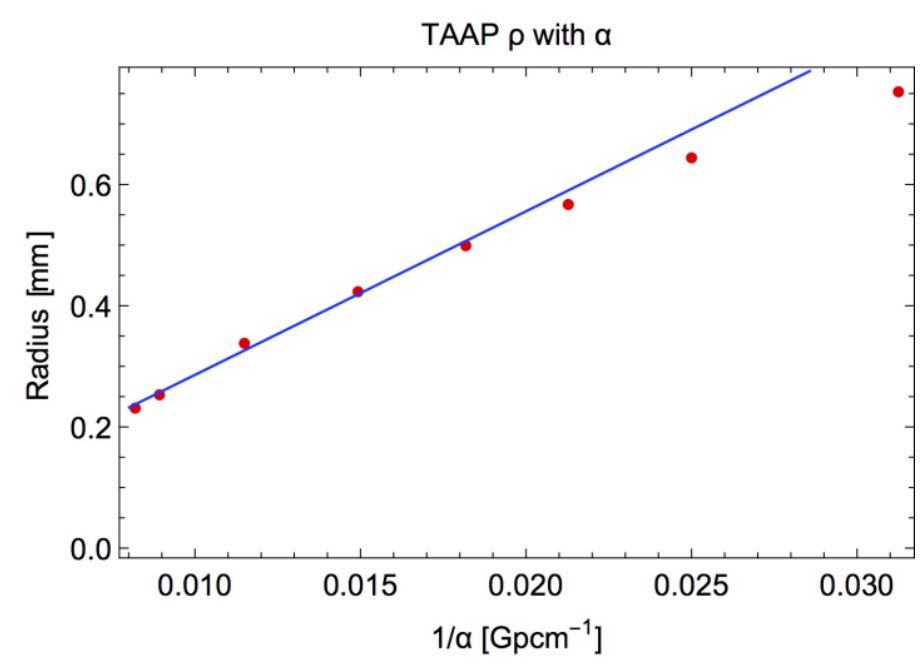

Figure 11. Radius of the ring vs. inverse quadrupole gradient $(\alpha)$ at a constant dressing frequency of $\omega_{\text {rf }} / 2 \pi=2.5 \mathrm{MHz}$. The data was taken with a dressing field, which was circularly polarized in the horizontal plane. The frequency and amplitude of the dressing field were kept constant. The solid line serves as guide to the eye demonstrating the deviation from the linear behavior, that is predicted for the case where the dressing field is modulated such that it stays resonant with the atoms on the ring.

The first step in the manipulation of the atoms around the ring should affect both spin states equally. For this we use gravity by tilting the modulation field with respect to the vertical axis, and thus tilt the ring. Gravity then pulls the atoms to one side of the ring. The trap-depth can be changed by adjusting the tilt-angle with respect to the vertical. By changing the azimuthal angle of the horizontal component of the modulation field one can place the atoms anywhere on the ring. 

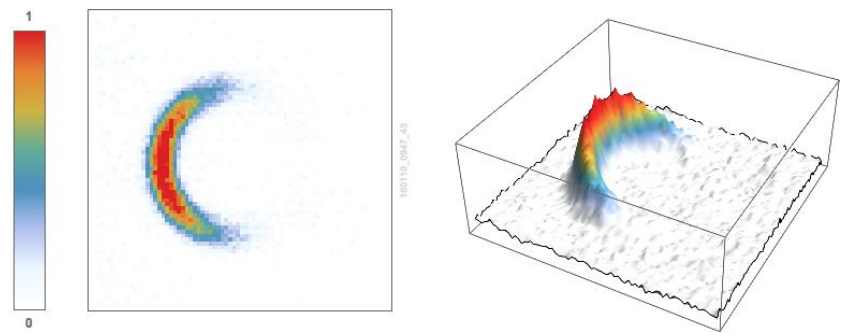

Figure 12. Experimental images of gravity tilted ring trap, where the position of the trap is identical for both spin states and is defined by the direction of the tilt. The imaged atoms are in $|2,+2\rangle$ state. The quadrupole gradient is $\alpha=55 \mathrm{G} / \mathrm{cm}$ and $\mathrm{rf}$ frequency is $\omega_{\mathrm{rr}} / 2 \pi=2.62 \mathrm{MHz}$. Radius of the ring is $490 \mu \mathrm{m}$ with $3 \times 10^{5}$ atoms in it.

The next important ingredient is the state dependent manipulation of the atoms: We start with the strong, linearly polarized rf-field in the z-direction and add a small rf-field in the horizontal plane, which has the same frequency as the first one but is 90 degrees out of phase with it. This creates a small component of circularly polarized rf. In the experiment of the figure below, we have realized coupling of atoms with a slightly elliptic polarized rf in the ring potential. The figure demonstrates trapping of $2 \times 10^{5}$ atoms in the $|1,-1\rangle$ state using a small circularly polarized $\mathrm{rf}$ component at about 30 degrees to the y-axis.
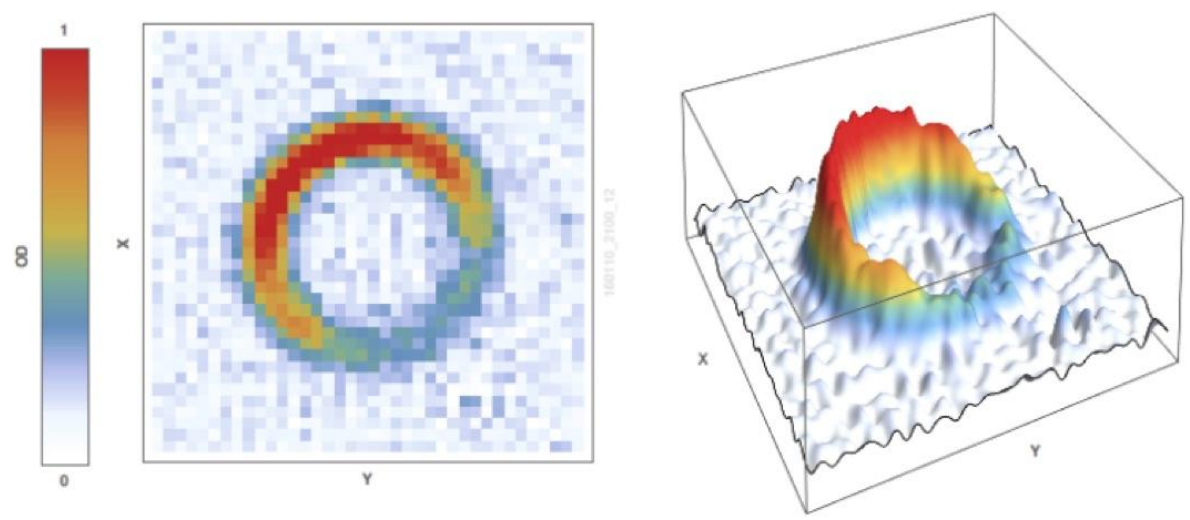

Figure 13. Experimental realization of state-dependent trapping, where the location of the trap is defined by the direction of the circular rf-component. The quadrupole gradient was $\alpha=55 \mathrm{Gpcm}$ and the dressing frequency $2.62 \mathrm{MHz}$. There are about $2 \times 10^{5}$ atoms in the of $|1,-1\rangle$ state at a temperature of a few micro Kelvin. The radius of the ring is $490 \mu \mathrm{m}$.

In order to demonstrate that we can move the atoms around the ring, we use a strong rf, which is circularly polarized in the $\mathrm{x}-\mathrm{y}$ plane and add a small linear rf, that is vertically polarized. This has the effect of creating a small $\mathrm{rf}$ component, which is circularly polarized in a plane containing the z-axes. By ramping the phase of the vertical rf component, we can then rotate this plane around the $\mathrm{z}$-axis. 


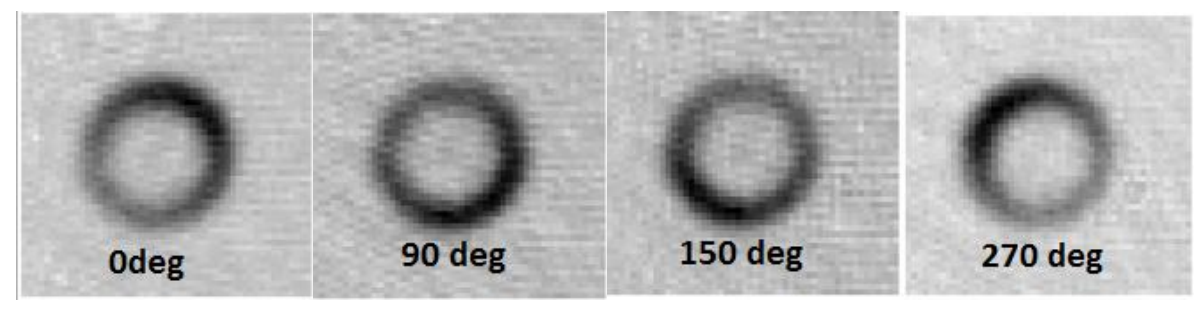

Figure 14. Guiding by a phase ramp. Number of atoms in the ring is $5.3 \times 10^{5}$ atoms in the in $|1,-1\rangle$ state. The dressing frequency is $2.5 \mathrm{MHz}$ at a quadrupole gradient of $70 \mathrm{G} / \mathrm{cm}$. Radius of the ring is $410 \mu \mathrm{m}$. The number

Finally, we can combine the state-dependent trap with the tilted one and create one or two traps, which we can place individually anywhere along the ring. In the final interferometric sequence we will pla
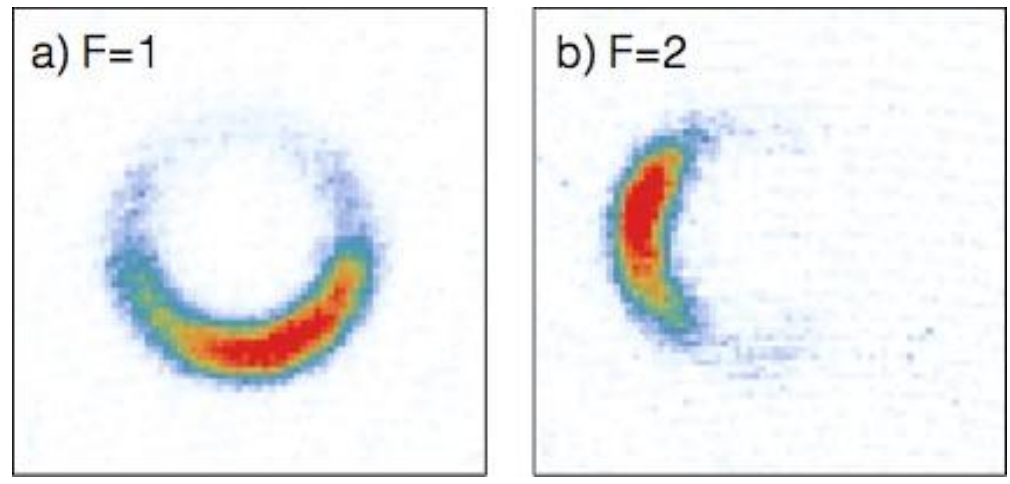

Figure $15 \mathrm{a}$ and $\mathrm{b}$ have identical experimental condition and differ only in the spin state of the atoms. In a) There are $5 \times 10^{5}$ atoms atoms in the $\left|\mathrm{F}, \mathrm{m}_{F}\right\rangle=|2,+2\rangle$ and in $\mathrm{b}$ ) there are $3 \times 10^{5}$ atoms in the $|1,-1\rangle$ state. The frequency of the dressing field was $\omega_{\mathrm{rf}} / 2 \pi=2.62 \mathrm{MHz}$. The fitted radiuses are $440 \mu \mathrm{m}$ and $450 \mu \mathrm{m}$ respectively. The quadrupole gradient was $\alpha=$ $55 \mathrm{G} / \mathrm{cm}$.

In conclusion: we have demonstrated the most important ingredient of the clock interferometer, that we can manipulate the atoms along the ring in a state dependent fashion.

We are now in the process of setting up the microwave beam splitters, which will allow us to complete the interferometric sequence.

\subsection{Atom detection for interferometer read-out}

The performance of the interferometer will also depend on the ability to perform accurate and precise read-out. Possible methods are absorption imaging, fluorescence detection or dispersive interaction. Radio-frequency dressed potentials introduce the possibility to detect atoms at high side-band frequencies in a dispersive detection scheme, which avoids low-frequency technical noise. Techniques that make use of such modulation of the atomic signal previously allowed for quantum noise limited performance and spin squeezing by quantum non-demolition measurements in large roomtemperature ensembles ${ }^{17}$. We have recently performed proof-of-principle experiments with radio-frequency dressed versions of standard clock states $\left(F_{z}=0\right)$ in rubidium-87. The principal setup is shown in Fig. 12. Atoms are released from a magneto-optical trap, optically pumped, state-purified and driven on a single photon microwave transition into superpositions of the two clock states. Subsequently, they are adiabatically transformed into RF dressed states and illuminated by off-resonant laser beams. A polarisation homodyne measurement is then used to determine the linear birefringence of the atomic sample, proportional to atom number. As the atomic spin state is locked to the effective field in the rotating frame, the rotation in the laboratory frame leads to phase-locked modulation of the balanced photodetector signal. The aligned but not-oriented character of the $\left|F_{z}=0\right\rangle$ states results in signal modulation at twice the dressing frequency. 


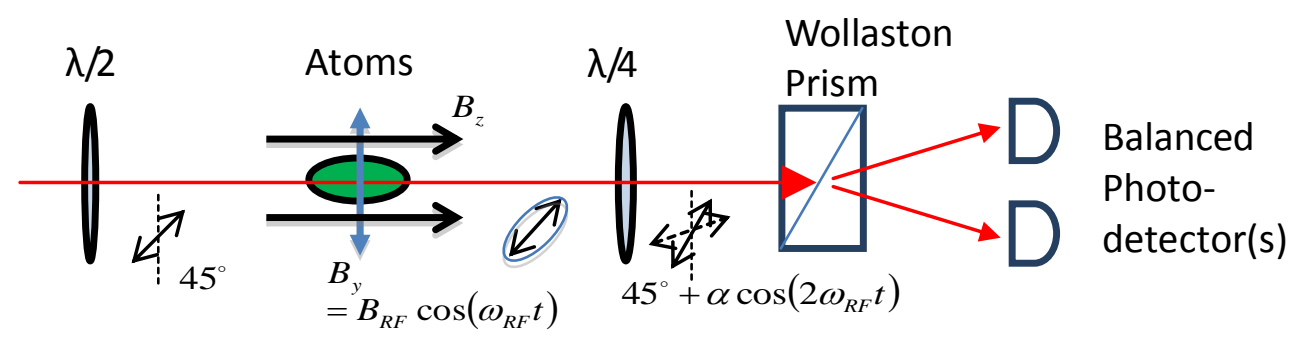

Figure 16. Setup for dispersive detection of radio-frequency dressed clock states.

We use a dressing frequency of $170 \mathrm{kHz}$ and phase-locked $340 \mathrm{kHz}$ detection with $10 \mathrm{kHz}$ bandwidth. By consecutively illuminating the atomic sample with two laser pulses of millisecond durations near the $F=1 \rightarrow F^{\prime}=1$ and $F=2 \rightarrow F^{\prime}=2$ transitions on the $\mathrm{D}_{1}$-line with approx. $300 \mathrm{MHz}$ detuning, we obtain separate signals for atoms in both $F=1$ and $F=2$, which allows for correction of shot-to-shot fluctuations of total atom number. The first results of such a measurement are presented in Fig. 13, showing a Rabi cycle of $10^{8}$ atoms with approx. $1 \%$ noise amplitude, limited by technical and light shot noise. This experiment was performed with laser beams that were not optimized for detuning, mode-overlap between atoms and probe beams, and relative intensity and frequency noise that lead to unbalanced signal variations from the two states. A similar effect stems from 50 Hertz noise in the static field direction, which was prominent at the resonant field of only $240 \mathrm{mG}$. With corresponding improvements, we expect to reach atomic shot noise limited performance.
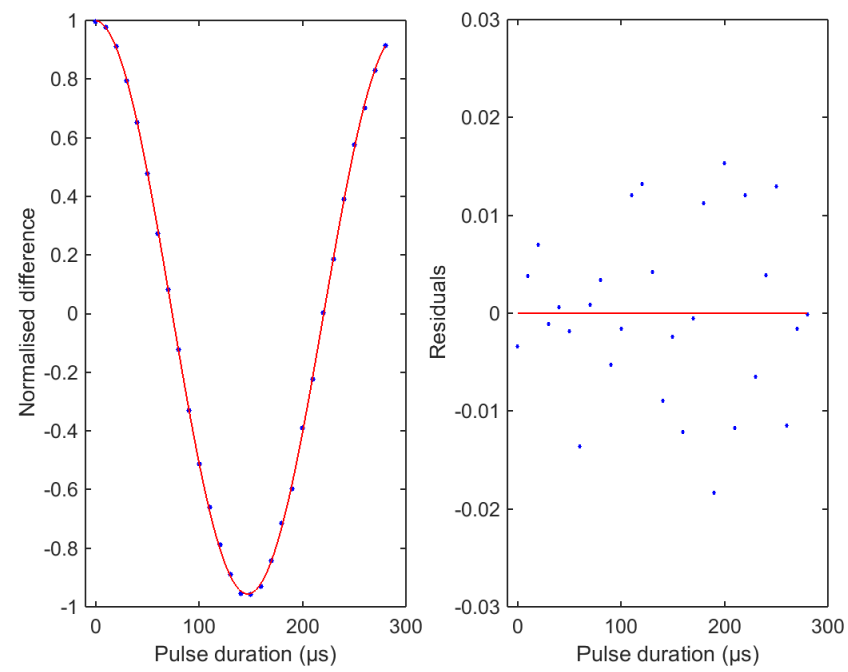

Figure 17. Experimental results from dispersive sideband detection of radio-frequency dressed clock states. The data shows population difference normalized to total atom number over a single cycle of a $3.5 \mathrm{kHz}$ Rabi oscillation together with residuals from the sinusoidal fit. The variance of the signal noise is approx. $10^{-4}$.

\section{CONCLUSIONS \& OUTLOOK}

We discussed the possibility to built compact Sagnac interferometers for matter waves using a scheme that is operated like an atomic clock and avoids the need for interferometric stability for optical beams. Recent experimental progress has been made on the required state-dependent guiding in time averaged adiabatic potentials. Two-state detection of radiofrequency dressed clock states has been demonstrated, which should allow for quantum noise limited performance of state read-out. We will concentrate future efforts on investigating effects that stem from the guided character of the interferometer and that may influence the achievable sensitivities. 


\section{ACKNOWLEDGEMENTS}

This research has also been supported by the EU-FET Grant FP7-ICT-601180 (MatterWave) and by FP7-PEOPLE-2012ITN- 317485 (QTea). TF, RS, MH, IL, TB, FG, SJ, TP and MB acknowledge support by the UK Engineering and Physical Sciences Research Council. We gratefully acknowledge useful discussions with the members of the MatterWave consortium.

\section{REFERENCES}

[1] Lawrence, A., [Modern Inertial Technology], Springer, New York (1998).

[2] Simonelli, A., Belfi, J., Beverini, N. Carelli, G. Di Virgilio, A., Maccioni, E., De Luca, G. and Saccorotti, G., "First deep underground observation of rotational signals from an earthquake at teleseismic distance using a large ring laser gyroscope," arXiv:1601.05960 (2016).

[3] Will, C. M., "The confrontation between general relativity and experiment," Living Reviews in Relativity 9, 3 (2006)

[4] Clauser, J. F., "Ultra-high sensitivity acceleromters and gyroscopes using neutral atom matter-wave interferometry," Physica B 151, 262 (1988).

[5] Barrett, B., Geiger, R., Dutta, I., Meunier, M., Canuel, B., Gauguet, A., Bouyer, P. and Landragin, A., "The Sagnac effect: 20 years of development in matter-wave interferometry," Comptes Rendus Physique 15, 875 (2014).

[6] Gustavson, T. L., Landragin, A. and Kasevich, M. A., "Rotation sensing with a dual atom-interferometer Sagnac gyroscope," Classical and Quantum Gravity 17(12), 2385 (2000).

[7] Durfee, D. S., Shaham, Y. K. and Kasevich, M. A., "Long-Term Stability of an Area-Reversible AtomInterferometer Sagnac Gyroscope,” Physical Review Letters 97(24), 240801 (2006).

[8] Hafele, J. C. and Keating, R. E., "Around-the-World Atomic Clocks: Observed Relativistic Time Gains," Science 177, 166 (1972).

[9] Fernholz, T., Gerritsma, R., Krüger, P. and Spreeuw, R. J. C., "Dynamically controlled toroidal and ring-shaped magnetic traps," Physical Review A 75(6), 063406 (2007).

[10] Stevenson, R., Hush, M. R., Bishop, T., Lesanovsky, I and Fernholz, T., "Rotation Sensing with a Single Atomic Clock," Physical Review Letters 115(16), 163001 (2015).

[11] Cohen-Tannoudji, C., Dupont-Roc, J. and Grynberg, G., [Atom-Photon Interactions], Wiley, New York, (1992).

[12] Bloch, F. and Siegert, A. J. F., "Magnetic Resonance for Nonrotating Fields," Phys. Rev. 57(6), 522 (1940).

[13] Treutlein, P, Hommelhoff, P. Steinmetz, T., Hänsch, T. W., Reichel, J., "Coherence in Microchip Traps," Physical Review Letters 92(20), 203005 (2004).

[14] Lesanovsky, I. and von Klitzing, W., "Time-Averaged Adiabatic Potentials: Versatile Matter-Wave Guides and Atom Traps," Physical Review Letters 99(8), 083001 (2007).

[15] Fernholz, T. Gerritsma, R., Whitlock, S., Barb, I. and Spreeuw, R. J. C., "Fully permanent magnet atom chip for Bose-Einstein condensation," Physical Review A 77(3), 033409 (2008).

[16] Morinaga, M., “Circular Magneto-Optical Trap for Neutral Atoms,” J. Phys. Soc. Japan 77, 104402 (2008).

[17] Sherson, J., Julsgaard, B. and Polzik, E. S., "Deterministic atom-light quantum interface," Advances in Atomic Molecular and Optical Physics 54, 81 (2006). 